\title{
Prenatal Nutritional Strategies to Reduce the Risk of Preterm Birth
}

\section{Karen Patricia Best ${ }^{a, b}$ Judith Gomersall ${ }^{a, c}$ Maria Makrides ${ }^{a, b}$}

aWomen and Kids Theme, South Australian Health and Medical Research Institute, Adelaide, SA, Australia; 'School of Medicine, University of Adelaide, Adelaide, SA, Australia; 'School of Public Health, University of Adelaide, Adelaide, SA, Australia

\section{Key Messages}

- Cost-effective primary prevention strategies to reduce preterm birth (PTB) are required to reduce the $\sim 15$ million preterm babies born every year worldwide. Nutritional interventions may offer a promising solution.

- The strongest evidence to date for a nutritional solution to reduce PTB exists for omega-3 long-chain polyunsaturated fatty acids and suggests that women with low levels of omega- 3 in early pregnancy may benefit from supplementation.

- Recent findings suggest that determining an individual woman's polyunsaturated fatty acid status in early pregnancy may be a precise way to inform recommendations to reduce her risk of PTB.

\section{Keywords}

Preterm birth · Nutrition · Pregnancy · Omega-3

Prevention · Supplementation · Prematurity

\begin{abstract}
Worldwide, around 15 million preterm babies are born annually, and despite intensive research, the specific mechanisms triggering preterm birth (PTB) remain unclear. Cost-effective primary prevention strategies to reduce PTB are required, and nutritional interventions offer a promising alternative. Nutrients contribute to a variety of mechanisms that are poten-
\end{abstract}

tially important to preterm delivery, such as infection, inflammation, oxidative stress, and muscle contractility. Several observational studies have explored the association between dietary nutrients and/or dietary patterns and PTB, often with contrasting results. Randomized trial evidence on the effects of supplementation with zinc, multiple micronutrients (iron and folic acid), and vitamin D is promising; however, results are inconsistent, and many studies are not adequately powered for outcomes of PTB. Large-scale clinical trials with PTB as the primary outcome are needed before any firm conclusions can be drawn for these nutrients. The strongest evidence to date for a nutritional solution exists for omega-3 long-chain polyunsaturated fatty acids (LCPUFAs), key nutrients in fish. In 2018, a Cochrane Review (including 70 studies) showed that prenatal supplementation with omega-3 LCPUFAs reduced the risk of PTB and early PTB (EPTB) compared with no omega- 3 supplementation. However, the largest trial of omega-3 supplementation in pregnancy, the Omega-3 to Reduce the Incidence of Prematurity (ORIP) trial ( $n=$ 5,544), showed no reduction in EPTB and a reduction in PTB only in a prespecified analysis of singleton pregnancies. Exploratory analyses from the ORIP trial found that women with low baseline total omega- 3 status were at higher risk of EPTB, and that this risk was substantially reduced with omega-3 supplementation. In contrast, women with replete or high baseline total omega- 3 status were already at low risk of EPTB karger@karger.com

www.karger.com/anm

C 2021 S. Karger AG, Basel

Karger $\stackrel{2}{*}$
Karen Patricia Best

Women and Kids, South Australian Health and Medical Research Institute 72 King William Road

North Adelaide, ADL 5006 (Australia)

karen.best @ sahmri.com 
and additional omega-3 supplementation increased the risk of EPTB compared to control. These findings suggest that determining an individual woman's PUFA status may be the most precise way to inform recommendations to reduce her risk of PTB.

(c) 2021 S. Karger AG, Basel

\section{Preterm Birth}

A human pregnancy usually lasts around 40 weeks, with most babies delivered at term (between 37 and 42 weeks of gestation; Fig. 1). Preterm birth (PTB) is defined by the World Health Organization (WHO) as all births before 37 completed weeks of gestation or fewer than 259 days since the first day of a woman's last menstrual period [1]. PTB is the second leading cause of death globally for children under 5 years of age [1]. It is estimated that $\sim 15$ million babies each year worldwide are born preterm with $20 \%$ occurring before 34 weeks gestation, referred to as early PTB (EPTB). EPTB is the major cause of perinatal mortality, serious neonatal morbidity, and moderate-to-severe childhood disability in developed countries [24]. These infants often require extended periods in hospital intensive care and may have developmental problems that can last a lifetime, including problems with their lungs, gut, and immune system function, in addition to problems with their vision and hearing. In early childhood, developmental difficulties may emerge, with later societal and economic impacts due to low educational achievement, high unemployment, and deficits in social and emotional well-being [5]. These outcomes have enormous economic and public health impact [6], and addressing PTB is an urgent priority.

\section{Preventing PTB, a Challenging Issue}

PTB is one of the most challenging issues in obstetric and neonatal care and is caused by multiple etiologies [7]. About half of the time, the causes of PTB are unclear, and there are no current satisfactory prevention strategies or treatments. Several Cochrane systematic reviews have been conducted on the effects of interventions designed to prevent PTB with treatments ranging from bed rest and smoking cessation to therapeutic drugs such as betamimetics, magnesium sulfate, and calcium channel blockers. While there has been some success in reducing the risk of PTB in high-risk women with tocolytic agents [8, 9], these are not suitable as prophylactic strategies because the risks associated with these interventions are not acceptable to the general population. One pharmacological intervention, which has been shown to be somewhat effective is progesterone, however, only in singleton pregnancies with a history of PTB [10]. In the absence of predictive tests that are sensitive, specific, and feasible to implement, more general cost-effective primary prevention strategies for PTB are required [7]. Nutritional interventions are promising alternatives.

\section{Maternal Nutrition}

Maternal nutrition before and during pregnancy plays an important role in providing the necessary nutrients for fetal growth [11] and may be a key factor in the risk of PTB [12]. Several observational studies have explored the association between dietary nutrients and PTB and present contrasting results. A cohort study in 60,000 women with singleton preg-

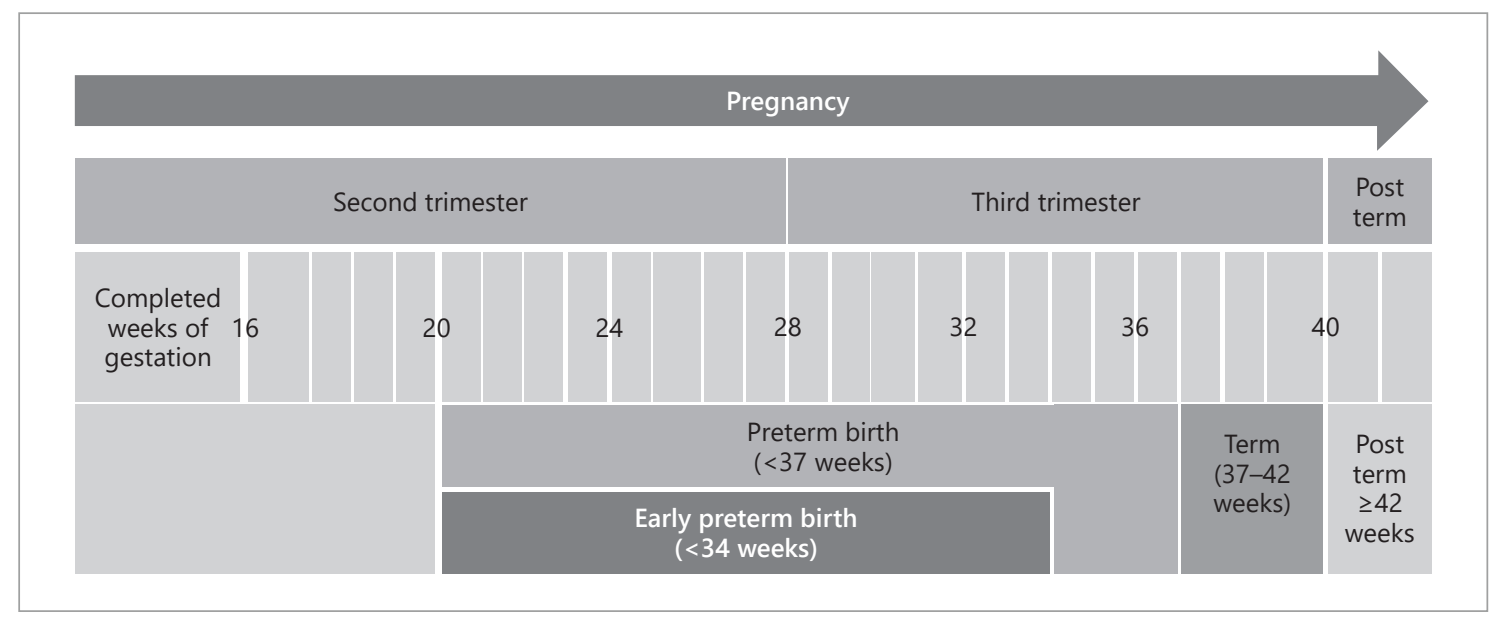

Fig. 1. Gestation at birth definitions. 
Table 1. Summary of Cochrane reviews assessing RCT evidence on effects of nutrients during pregnancy on PTB and EPTB

Magnesium versus no magnesium and PTB [22]

Calcium versus placebo/no treatment and EPTB [23]

Calcium versus placebo/no treatment and PTB [23]

Iron alone versus placebo/no treatment and PTB [24]

Folic acid alone versus placebo/no treatment and PTB [25]

Iron and folic acid versus placebo/no treatment and PTB [24]

Supplements containing iron and folic acid versus same supplements without iron

nor folic acid or placebo and PTB [24]

MMN (with iron and folic acid) versus iron with or without folic acid and PTB [26]

Zinc alone or in combination with other micronutrients versus placebo and PTB [29]

Vitamin D alone versus placebo/no treatment and PTB [27]

Vitamin D and calcium versus placebo/no treatment and PTB [27]

Omega-3 LCPUFA compared with no omega-3 and PTB [28]

Omega-3 LCPUFA compared with no omega-3 and EPTB [28]
7 trials, 5,981 women RR 0.89 (95\% Cl 0.69-1.14)

4 trials, 5,669 women RR 1.04 (95\% Cl 0.8-1.36)

13 trials; 16,139 women RR 0.86 (95\% Cl 0.7-1.05)

6 trials, 1,713 women $\quad$ RR $0.82(95 \% \mathrm{Cl} 0.58-1.14)$

3 trials, 2,959 women RR 1.01 (95\% Cl 0.73-1.38)

3 trials, 1,479 women RR 1.55 (95\% Cl 0-4.6)

3 trials, 1,497 women $\quad$ RR $1.55(95 \%$ Cl 0.40-6.00)

8 trials, 91,425 women RR 0.95 (95\% Cl 0.90-1.01)

16 trials, 7,637 women $\quad$ RR $0.86(95 \% \mathrm{Cl} 0.76-0.97)$

7 trials, 1,640 women $\quad$ RR $0.66(95 \% \mathrm{Cl} 0.34-1.30)$

5 trials, 942 women RR $1.52(95 \% \mathrm{Cl} 1.01-2.28)$

26 trials, 10,304 women RR 0.89 (95\% Cl 0.81-0.97)

9 trials, 5,204 women RR $0.58(95 \% \mathrm{Cl} 0.44-0.77)$
Not applicable ${ }^{a}$ Moderate

Moderate

Not applicable

Not applicable ${ }^{a}$

Not applicable

Low

Moderate

Moderate

Low certainty

Low certainty

High

High

EPTB, early preterm birth (<34 weeks); LCPUFA, long-chain polyunsaturated fatty acid; PTB, preterm birth ( $<37$ weeks): RCT, randomized controlled trial; MD, mean difference; MMN, multiple micronutrients; RR, relative risk. ${ }^{a}$ Quality of evidence not assessed in the review.

nancies in Norway observed an association between higher intake of artificially sweetened and sugar-sweetened beverages and increased risk of PTB [13]. Another study based on the same pregnancy cohort assessed the risk of PTB for 3 dietary patterns: "prudent" (vegetables, fruits, oils, water as beverage, whole grain cereals, and fiber-rich bread), "Western" (salty and sweet snacks, white bread, desserts, and processed meat products), and "traditional" (potatoes and fish), reporting that high scores on the "prudent" dietary pattern were associated with significantly reduced risk of PTB (hazard ratio for the highest vs. the lowest third: $0.88,95 \% \mathrm{Cl} 0.80-0.97)$. The "traditional" diet was associated with reduced risk of PTB for the highest versus the lowest third (hazard ratio 0.91, 0.83-0.99), and no independent association with PTB was found for the "Western" diet [14]. Another cohort study, in Denmark (Danish National Birth Cohort), showed consumption of a Mediterranean diet in mid-pregnancy (including fish at least bi-weekly, using olive or grape seed oil, $>5$ portions of fruit and vegetables/day, meat no more than twice a week, and at most 2 cups of coffee/day) was associated with a $72 \%$ lower risk of EPTB [15].

The epidemiological evidence on dietary patterns and PTB has been summarized in 3 recent systematic reviews [16-18]. The review by Chia and colleagues [16], a meta-analysis including 6 studies, showed adherence to a "healthy" diet comprising high intakes of vegetables, fruits, whole grains, low-fat dairy, and lean protein foods lowered the risk of PTB lodds ratio $[\mathrm{OR}]$ for the top compared to bottom tertile $[0.79,95 \%$ Cl 0.68-0.91]). Kibret et al. [17] pooled data from 9 studies to assess the association between adherence to a "healthy dietary pattern" defined as high intake of vegetables, fruits, whole grain foods, poultry and fish, and PTB. Reduced odds of PTB were observed (OR 0.75, 95\% Cl 0.57-0.93), although there was significant heterogeneity between studies $\left(I^{2}=\right.$ $89.6 \%$ ). Analysis based on 4 of the studies presented in this review showed a "Western" diet, comprising mostly refined grains, processed meats or snacks, high-sugar and high-fat dairy products, eggs, and white potatoes had no effect on the odds of PTB (OR 1.11, 95\% Cl 0.87-1.34), though again substantial heterogeneity was seen $\left(I^{2}=77.8 \%\right)$ [17]. Raghavan and colleagues [18] performed a narrative review of the evidence for associations between dietary patterns during pregnancy and PTB. They concluded that although the evidence is limited, there is some evidence (mostly studies involving Caucasian women) to support protective associations with PTB. These protective dietary patterns are higher in vegetables; fruits; whole grains; nuts; legumes and seeds; and seafood, and lower in red and processed meats and fried foods [18].

Fish is a rich source of essential nutrients for fetal development which has been linked to a reduction in PTB since the 1980s when it was noticed that women who ate a lot of fish in the Faroe Islands (in Scandinavia) had longer pregnancies than their Danish neighbors [19]. A systematic review by Leventakou et al. [20] assessed the evidence for associations between fish intake and PTB, adjusting for a wide range of potentially important confounding variables in all meta-analyses. A total of 19 population-based European birth cohort studies and 151,880 mother-child pairs were included in this 
Fig. 2. Synthesis of polyunsaturated fatty acids.

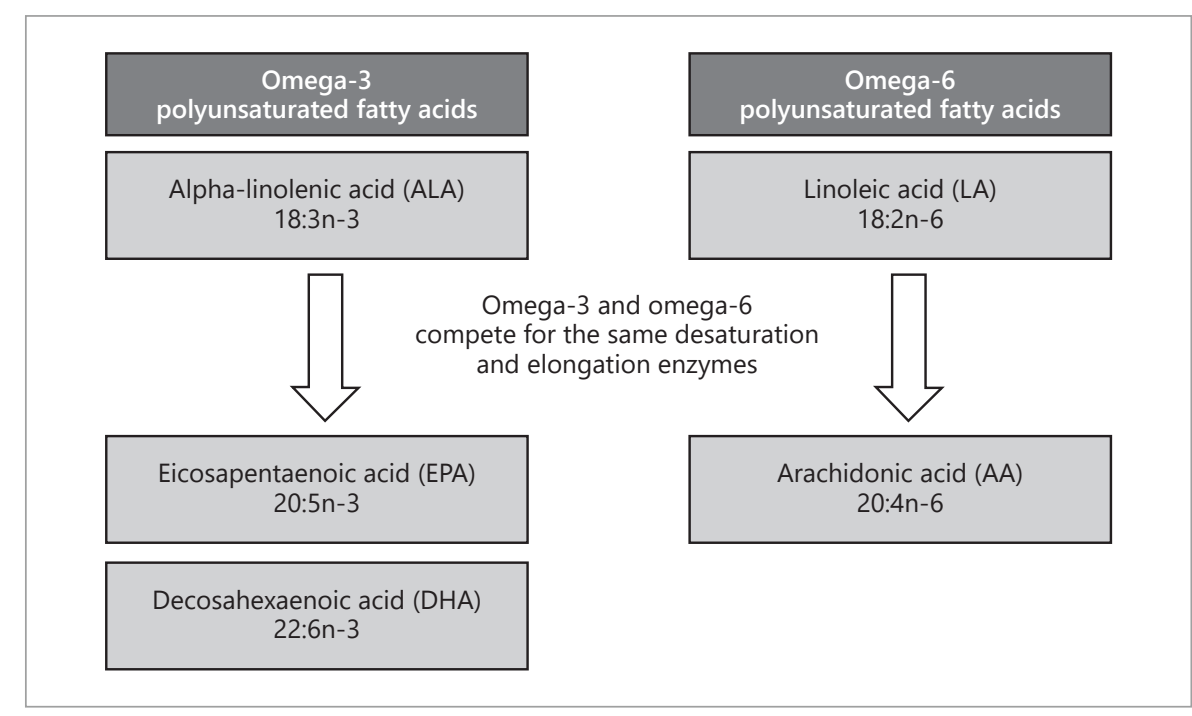

review. Findings were consistent across cohorts; the adjusted RR of fish intake $>1$ but $<3$ times/week compared to $\leq 1$ time/ week was 0.87 (95\% Cl 0.82-0.92) and of fish intake $\geq 3$ times/ week compared to $\leq 1$ time/week was $0.89(95 \% \mathrm{Cl} 0.84-$ 0.96). This large international study indicates that moderate fish intake during pregnancy is associated with lower risk of PTB [20]. Although some conclusions regarding dietary patterns and fish intake can be drawn from these reviews, interpretation is difficult due to the methodological limitations of epidemiological studies and the risk of bias from residual confounding [21].

Randomized controlled trials (RCTs) are the most reliable type of research to inform questions about cause and effect. A substantial body of RCT evidence on the effects of supplementation with individual nutrients, including magnesium, calcium, iron, folic acid, zinc, vitamin D, omega-3, or multiple micronutrients (MMNs), on PTB has accumulated. Samuel et al. [7] have provided a systematic overview of the evidence on nutritional solutions for PTB risk reduction, and various Cochrane reviews [22-28] assess the RCT evidence on the effects of these nutrients on PTB (Table 1). A review of magnesium supplementation during pregnancy showed no difference in risk of PTB between women who received magnesium versus no magnesium [22]. Another review found moderate quality evidence indicating no reduction in PTB or EPTB risk between women who received calcium during pregnancy compared to placebo or no treatment [23]. Two early Cochrane reviews found no differences in PTB between women who received iron alone compared with no treatment/placebo [24]; folic acid alone compared with no treatment or placebo [25]; daily iron and folic acid supplements versus no treatment/placebo [24]; or any supplements containing iron and folic acid compared with the same supplements without iron nor folic acid or placebo [24]. More recently, a Cochrane review evaluating benefits of MMNs supplementation with iron and folic acid during pregnancy found moderate quality evidence for a small effect of MMNs (with iron and folic acid) compared to iron with or without folic acid on the risk of PTB [26].

There is some limited evidence for administration of zinc supplements (5-44 mg/day) as well as vitamin D supplementation as potentially effective interventions to prevent PTB [7]. A Cochrane review demonstrated moderate quality evidence of a small but significant $14 \%$ reduction in PTB with antenatal supplementation of zinc alone or in combination with other micronutrients compared to placebo [29]. However, most of the RCTs assessing zinc have been conducted in low-income countries among women with poor nutritional status, likely to have had low zinc concentrations. The reduction in PTB observed in these studies has not been accompanied by a reduction in LBW or a difference in gestational age at birth, suggesting that it is too early to be certain about the beneficial effects of zinc [7]. Vitamin D deficiency in women of reproductive age is widespread, and low maternal vitamin D status during pregnancy is a risk factor for several adverse birth outcomes including PTB [30]. A recent review by De-Regil et al. [27] found low-quality evidence showing no difference in PTB between women who received vitamin $\mathrm{D}$ alone compared to placebo or no treatment, or between women who received vitamin $D$ and calcium versus placebo or no treatment during pregnan$c y$. The only high-quality evidence to date for a nutritional solution to prevent PTB (in singleton pregnancies) exists for omega-3 long-chain polyunsaturated fatty acids (LCPUFAs). 
Table 2. Summary of 2018 Cochrane review of marine oil supplementation in pregnancy outcomes [35]

\begin{tabular}{|c|c|c|c|}
\hline \multirow{2}{*}{$\begin{array}{l}\text { Variable } \\
\text { Birth <34 weeks }\end{array}$} & \multicolumn{2}{|c|}{ Effect of omega-3 LCPUFA treatment relative to control } & \multirow{2}{*}{$\begin{array}{l}\text { Quality of the } \\
\text { evidence (grade) } \\
\text { High }\end{array}$} \\
\hline & 11 trials, 5,409 women & RR $0.58(95 \% \mathrm{Cl} 0.44-0.77)$ & \\
\hline Birth $<37$ weeks & 25 trials, 10,256 women & RR $0.89(95 \%$ Cl $0.81-0.97)$ & High \\
\hline Birth $>42$ weeks & 6 trials with 5,141 women & RR $1.61(95 \% \mathrm{Cl} 1.11-2.33)$ & Moderate \\
\hline Gestational length & 41 trials with 12,517 women & MD 1.67 days $(0.95-2.39)$ & Moderate \\
\hline Preeclampsia ${ }^{a}$ & 20 trials with 8,306 women & RR $0.84(0.69-1.01)$ & Low \\
\hline Perinatal death & 10 trials with 7,416 women & RR $0.75(0.54-1.03)$ & Moderate \\
\hline Birth weight, g & 42 trials with 11,584 women & MD $76 \mathrm{~g}$ higher (38 to 113 higher) & Not applicable ${ }^{b}$ \\
\hline Low birth weight $<2,500 \mathrm{~g}$ & 15 trials with 8,449 women & RR $0.90(0.82-0.99)$ & High \\
\hline SGA & 8 trials with 6,907 women & RR $1.01(0.90-1.13)$ & Moderate \\
\hline LGA & & RR $1.15(0.97-1.36)$ & Moderate \\
\hline
\end{tabular}

LGA, large for gestational age; LCPUFA, long-chain polyunsaturated fatty acid; MD, mean difference; RR, relative risk; SGA, small for gestational age. ${ }^{a}$ Defined as hypertension with proteinuria. ${ }^{b}$ Quality of evidence for this outcome not assessed in the review.

\section{Omega-3 and PTB}

Omega-3 is an essential fatty acid which must be obtained from the diet and is a key nutrient in fish. First observed in the 1980s and recently supported in the large Leventakou review, long-chain omega-3 fatty acids from marine sources such as fish and algae are thought to be responsible for longer pregnancies (and fewer preterm babies) $[19,20]$. There are plausible biological mechanisms to indicate that dietary insufficiency of omega-3 LCPUFA may play a role in the pathophysiology of preterm delivery, and this presents a potential target for intervention. Prostaglandins and other oxylipins derived from omega -6 and omega- 3 fatty acids play essential roles in normal and pathologic initiation of labor [31, 32]. The fetoplacental unit is supplied with LCPUFAs from the maternal circulation, which is influenced by maternal LCPUFA intake and endogenous synthesis (Fig. 2). The prostaglandins and oxylipins derived from omega- 6 arachidonic acid within the utero-placental unit in normal pregnancy are countered by local production of prostaglandins and oxylipins derived from omega-3 LCPUFA within the same tissues. The balance between the metabolites of omega- 3 and omega- 6 fatty acids plays a vital role in the maintenance of normal gestational length and is a critical element in cervical ripening and the initiation of labor $[33,34]$. If local production of omega- 6 -derived prostaglandins within the feto-placental unit is too high, or local accumulation of omega-3 LCPUFA is too low, the cervix may prematurely ripen and uterine contractions increase, which may in turn lead to PTB.

Current Western diets are low in omega-3 LCPUFAs and high in omega- 6 fatty acids. The WHO recommends an intake of $300 \mathrm{mg}$ of omega-3 LCPUFAs per day in pregnant women; however, the median intake among Australian and American women of childbearing age is less than one-third of this, compared with $1,000 \mathrm{mg} /$ day in many women from nations with high fish consumption, such as Japan, Korea, and Norway [35]. Several epidemiological studies and RCTs have investigated the effect of increased maternal omega-3 intake and PTB. Middleton et al. [28] recently updated the Cochrane Review of Marine Oil Supplementation in Pregnancy, which was first published in 2006 [36]. This updated review includes all trials of LCPUFAs in any form or dose during pregnancy (including as supplements, food, or dietary advice). The latest search of the literature was conducted in August 2018. The review included 70 RCTs, involving 19,927 women. Most of the trials were conducted in high-income countries (e.g., USA, England, The Netherlands, Australia, and Denmark), and most included women were carrying singleton pregnancies. The intervention dose ranged between 200 and 2,700 mg omega-3 LCPUFA as docosahexaenoic acid (DHA) or eicosapentaenoic acid (EPA) and was administered mainly throughout the second half of pregnancy. Results show high-quality evidence that supplementation with omega-3 LCPUFA during pregnancy reduced the risk of having a premature baby $<37$ weeks' gestation by $11 \%$ and $<34$ weeks' gestation by $42 \%$ compared with no omega- 3 supplementation. Additional outcomes for the systematic reviewed showed that prenatal omega-3 LCPUFA supplementation was safe (in terms of no effect on bleeding or postpartum hemorrhage) and significantly reduced the incidence of low birth weight and increased the incidence of pregnancies continuing beyond 42 weeks, although there was no difference identified in induction of labor for post-term pregnancies (Table 2). 
Table 3. Summary of the ORIP trial outcomes [39]

\begin{tabular}{lccl}
\hline Variable & $\begin{array}{l}\text { Omega-3 group } \\
(n=2,734), n(\%)\end{array}$ & $\begin{array}{l}\text { Control group } \\
(n=2,752), n(\%)\end{array}$ & $\begin{array}{l}\text { Adjusted RR } \\
(95 \% \mathrm{Cl})^{\mathrm{a}}\end{array}$ \\
\hline Birth <34 weeks & $61(2.2)$ & $55(2.0)$ & $1.13(0.79-1.63)$ \\
Birth <37 weeks & $211(7.7)$ & $246(8.9)$ & $0.86(0.72-1.03)$ \\
Prolonged gestation & $12(0.4)$ & $12(0.4)$ & N/A \\
Gestational length, days & $273.2 \pm 15.2$ & $273.2 \pm 14.9$ & $0.02(-0.78$ to 0.82) \\
Preeclampsia & $96(3.5)$ & $91(3.3)$ & $1.07(0.80-1.43)$ \\
Perinatal death & $32(1.1)$ & $25(0.9)$ & $1.28(0.76-2.17)$ \\
Birth weight, g & $3,351 \pm 628$ & $3,340 \pm 591$ & $10.56(-23.87$ to 44.99$)$ \\
Low birth weight $<2,500 \mathrm{~g}$ & $204 / 2,787(7.3)$ & $173 / 2,800(6.2)$ & $1.18(0.95-1.47)$ \\
SGA & $206 / 2,787(7.4)$ & $196 / 2,800(7.0)$ & $1.06(0.87-1.28)$ \\
LGA & $392 / 2,787(14.1)$ & $355 / 2,800(12.7)$ & $1.11(0.97-1.27)$ \\
\hline
\end{tabular}

g, grams; LGA, large for gestational age; LCPUFA, long-chain polyunsaturated fatty acid; MD, mean difference; RR, relative risk; SGA, small for gestational age; ORIP, Omega-3 to Reduce the Incidence of Prematurity. ${ }^{a}$ The effect sizes are relative risks (omega-3 group vs. control group) unless otherwise indicated. The adjusted values were adjusted for randomization strata: recruitment hospital and consumption of dietary supplements containing $n-3$ LCPUFA in the previous 3 months (yes or no). Except in the case of the primary outcome, the $95 \% \mathrm{Cl}$ were not adjusted for multiplicity and therefore should not be used to infer treatment effects. ${ }^{b}$ The effect size is the difference in means (omega-3 group minus control group).

It is important to note that this latest Cochrane review includes studies mainly from high-income countries with lowrisk, normal-risk, and high-risk women, and almost all women had singleton pregnancies. Reporting biases may underestimate or overestimate omega-3 effects on prematurity and other adverse birth outcomes and further work is needed to address concerns that supplementation in late pregnancy may prolong gestation beyond term. For example, the 2-day shift in mean gestation in our DOMInO trial (the largest trial included in the systematic review with 2,499 women) increased the number of post-term pregnancies and thus the need for more obstetric interventions to initiate birth (17.6 vs. 13.7\%; RR 1.28, 95\% Cl 1.06-1.54) [37]. This highlighted the need for further research to investigate the effects of prenatal omega-3 supplementation in a broad representation of women before adopting a universal supplementation approach into routine antenatal care.

The Omega- 3 to Reduce the Incidence of Prematurity (ORIP) RCT of 5,544 pregnancies was published in 2019 [38]. It is the largest trial to assess whether omega-3 LCPUFA supplementation, mainly as DHA, reduced the risk of EPTB (<34 weeks' gestation) [39]. This trial was designed with the unique feature of ceasing the intervention at 34 weeks' gestation (when the risk of EPTB has passed) to avoid prolongation of pregnancy requiring post-term obstetric intervention. The ORIP trial was specifically designed to assess a broad-based supplementation strategy inclusive of single- ton and multiple pregnancies, inclusive of women regardless of prematurity risk, and inclusive of many women already taking low-dose omega-3 supplements. This contrasts with most other studies that included only singleton pregnancies and/or focused on women with low intakes. This heterogeneity may, in part, explain why the results of the ORIP trial are discordant with those of the systematic review. The ORIP trial found that supplementation of pregnant women with $900 \mathrm{mg}$ per day of omega-3 LCPUFA (800 $\mathrm{mg}$ DHA/100 mg EPA) did not reduce the overall risk of EPTB or PTB, even though omega-3 PUFA concentrations in the intervention group at 34 weeks' gestation were elevated relative to the control group (Table 3 ).

Prespecified secondary analyses of only singleton pregnancies in the ORIP trial suggested a reduction in the risk of PTB with omega- 3 supplementation in singleton but not multiple pregnancies (RR 0.81, 95\% Cl 0.67, 0.99) [39]. The ORIP trial also comprised the valuable inclusion of blood samples to determine maternal baseline omega-3 status prior to commencing supplementation (trial entry $<20$ weeks' gestation), a design feature most of the prior trials lack. Exploratory analyses in women with a singleton pregnancy $(n=5,070)$ found that women with low baseline total omega- 3 blood PUFA status $(<4.1 \%, n=885)$ were at higher risk of EPTB and that this risk was substantially reduced by $77 \%$ with omega-3 supplementation (relative risk $=0.23,95 \% \mathrm{Cl} 0.07-0.79$ ) [40]. In contrast, women with higher or replete total omega-3 status at 
baseline $(>4.9 \%, n=2,277)$ were at lower risk of EPTB, and supplementing these women with omega- 3 increased the risk of EPTB compared to control (relative risk $=2.27$, 95\% $\mathrm{Cl} 1.13-$ 4.58) [40].

This observation that EPTB is altered by baseline omega-3 status is consistent with epidemiological data showing that low fish intake [20] or low omega-3 status [41] is associated with an increased risk of EPTB in singleton pregnancies. In a case-control study nested within the Danish National Birth Cohort, 376 EPTB cases were identified. When comparing concentrations of EPA plus DHA, women in the lowest quintile of the distribution had a 10 times $(95 \% \mathrm{Cl} 6.8-16, p<0.0001)$ increased risk of EPTB, and women in the second lowest quintile had a 2.9 times $(95 \% \mathrm{Cl} 1.8-4.6, p<0.0001)$ increased risk of EPTB, when compared to women in the 3 aggregated highest quintiles [41]. A moderate fish intake during pregnancy (1-2 fish meals per week) would generally be associated with a total omega -3 status of $>4.1 \%$ of total omega -3 fatty acids in whole blood, the conservative cutoff reported in the ORIP exploratory analysis showing a protective association with EPTB [40].

The unexpected findings from the ORIP exploratory analysis suggesting an increased risk of EPTB in women with replete omega-3 status have been proposed previously. Klebanoff et al. [42] conducted a secondary analysis of a prenatal omega-3 supplementation RCT and report that for women at risk of recurrent PTB, the probability of PTB was highest at low and high intakes, and lowest with moderate fish consumption. Similar patterns have been seen for several micronutrients and higher risks of adverse health outcomes for both low and high nutrient intakes - a U-shaped relationship [43]. Determining a woman's omega-3 status in early pregnancy and likelihood of benefiting from omega-3 supplementation to reduce her risk of EPTB would be the most precise way to inform supplementation practices. We would recommend that women with replete omega-3 status in early pregnancy should continue their current dietary practices to maintain their status. However, correcting low maternal omega-3 status through supplementation may reduce her risk of EPTB.

\section{References}

1 March of dimes, the partnership for maternal, newborn and child health, save the children, WHO. Born too soon: the global action report on preterm birth. Geneva, Switzerland: World Health Organisation; 2012.

2 Lumley J. Defining the problem: the epidemiology of preterm birth. BJOG. 2003;110(Suppl 20):3-7.

3 Martin JAOM. Describing the increase in preterm births in the United States, 2014-2016. In: NCHS, editor. Data Brief, no 312 ed. Hyattsville, MD: National Center for Health Statistics; 2018.

\section{Summary}

Despite intensive research, the mechanisms triggering the 15 million PTBs occurring worldwide every year remain unclear. Nutritional interventions are promising primary prevention strategies, yet to date, many broad-based interventions with the potential to reduce the risk of PTB are effective only in specific groups of women, most likely due to the heterogeneity of the population and the etiopathogenesis of PTB. At present, omega-3 PUFA seems to be the nutrient holding the most promise for the prevention of EPTB. Based on the available evidence, omega-3 supplementation during pregnancy to prevent EPTB should be targeted to women with low omega-3 status in early pregnancy. Clinicians should discuss the importance of a good diet with pregnant women and in the absence of measured maternal omega-3 PUFA levels, advise dietary source essential fatty acids be regularly consumed during pregnancy, and low-dose fish oil supplements may be explored to provide the necessary omega-3 required for optimal maternal and fetal outcomes. Advancement of this field requires the development and implementation of a targeted approach and evidence-based precision nutrition in antenatal care [7].

\section{Statement of Ethics}

No approval was required for this review.

\section{Conflict of Interest Statement}

The writing of this article was supported by Nestlé Nutrition Institute. Professor Makrides serves at the Board of Directors for Trajan Nutrition. Dr. Best and Dr. Gomersall have nothing to disclose.

\section{Funding Sources}

Dr. Best is supported by a MS McLeod Post-Doctoral Fellowship. Professor Makrides is supported by a National Health and Medical Research Fellowship.

4 Pretorius C, Jagatt A, Lamont RF. The relationship between periodontal disease, bacterial vaginosis, and preterm birth. J Perinat Med. 2007;35(2):93-9.

5 Westrupp E, Lucas N, Mensah F, Gold L, Wake M, Nicholson JM. Community-based healthcare costs for children born low birthweight, preterm and/or small for gestational age: data from the Longitudinal Study of Australian Children. Child Care Health Dev. 2014; 40(2):259-66 
6 Blencowe H, Cousens S, Oestergaard MZ, Chou D, Moller AB, Narwal $\mathrm{R}$, et al. National, regional, and worldwide estimates of preterm birth rates in the year 2010 with time trends since 1990 for selected countries: a systematic analysis and implications. Lancet. 2012;379(9832):2162-72.

7 Samuel TM, Sakwinska O, Makinen K, Burdge GC, Godfrey KM, Silva-Zolezzi I. Preterm birth: a narrative review of the current evidence on nutritional and bioactive solutions for risk reduction. Nutrients. 2019;11(8):1811.

8 Dodd JM, Flenady VJ, Cincotta R, Crowther CA. Progesterone for the prevention of preterm birth: a systematic review. Obstet Gynecol. 2008;112(1):127-34.

9 King JF, Flenady V, Papatsonis D, Dekker G, Carbonne B. Calcium channel blockers for inhibiting preterm labour; a systematic review of the evidence and a protocol for administration of nifedipine. Aust N Z J Obstet Gynaecol. 2003;43(3):192.

10 Romero R, Nicolaides KH, Conde-Agudelo A, O'Brien JM, Cetingoz E, Da Fonseca $E$, et al. Vaginal progesterone decreases preterm birth $</=34$ weeks of gestation in women with a singleton pregnancy and a short cervix: an updated meta-analysis including data from the OPPTIMUM study. Ultrasound Obstet Gynecol. 2016;48(3):308-17.

11 Nnam NM. Improving maternal nutrition for better pregnancy outcomes. Proc Nutr Soc. 2015;74(4):454-9.

12 Fuchs F, Senat MV. Multiple gestations and preterm birth. Semin Fetal Neonatal Med. 2016;21(2):113-20.

13 Englund-Ögge L, Brantsæter AL, Haugen M, Sengpiel V, Khatibi A, Myhre R, et al. Association between intake of artificially sweetened and sugar-sweetened beverages and preterm delivery: a large prospective cohort study. Am J Clin Nutr. 2012;96(3):552-9.

14 Englund-Ögge L, Brantsæter AL, Sengpiel V, Haugen M, Birgisdottir BE, Myhre R, et al. Maternal dietary patterns and preterm delivery: results from large prospective cohort study. BMJ. 2014;348: g1446.

15 Mikkelsen TB, Osterdal ML, Knudsen VK, Haugen M, Meltzer HM, Bakketeig $\mathrm{L}$, et al. Association between a Mediterranean-type diet and risk of preterm birth among Danish women: a prospective cohort study. Acta Obstet Gynecol Scand. 2008;87(3):325-30.

16 Chia A-R, Chen L-W, Lai JS, Wong CH, Neelakantan N, van Dam RM, et al. Maternal dietary patterns and birth outcomes. a systematic review and meta-analysis. Adv Nutr. 2019 Jul 1;10(4):685-95.

17 Kibret KT, Chojenta C, Gresham E, Tegegne TK, Loxton DJP. Maternal dietary patterns and risk of adverse pregnancy (hypertensive disorders of pregnancy and gestational diabetes mellitus) and birth (preterm birth and low birth weight) outcomes: a systematic review and meta-analysis. Public Health Nutr. 2019;22(3):506-20.

18 Raghavan R, Dreibelbis C, Kingshipp BL, Wong YP, Abrams B, Gernand $A D$, et al. Dietary patterns before and during pregnancy and maternal outcomes: a systematic review. Am J Clin Nutr. 2019; 109(Suppl 7):705S-28S.

19 Olsen SF, Hansen HS, Sørensen TI, Jensen B, Secher NJ, Sommer $S$, et al. Intake of marine fat, rich in (n-3)-polyunsaturated fatty acids, may increase birthweight by prolonging gestation. Lancet. 1986;2(8503):367-9.
20 Leventakou V, Roumeliotaki T, Martinez D, Barros H, Brantsaeter $\mathrm{AL}$, Casas $\mathrm{M}$, et al. Fish intake during pregnancy, fetal growth, and gestational length in 19 European birth cohort studies. Am J Clin Nutr. 2014;99(3):506-16.

21 Khoury J, Henriksen T, Christophersen B, Tonstad S. Effect of a cholesterol-lowering diet on maternal, cord, and neonatal lipids, and pregnancy outcome: a randomized clinical trial. Am J Obstet Gynecol. 2005;193(4):1292-301.

22 Makrides M, Crosby DD, Bain E, Crowther CA. Magnesium supplementation in pregnancy. Cochrane Database Syst Rev. 2014 Apr 3;2014(4):CD000937.

23 Buppasiri P, Lumbiganon P, Thinkhamrop J, Ngamjarus C, Laopaiboon M, Medley N. Calcium supplementation (other than for preventing or treating hypertension) for improving pregnancy and infant outcomes. Cochrane Database Syst Rev. 2015 Feb;25(2): CD007079.

24 Pena-Rosas JP, De-Regil LM, Garcia-Casal MN, Dowswell T. Daily oral iron supplementation during pregnancy. Cochrane Database Syst Rev. 2015 Jul 22;(7):CD004736

25 Lassi ZS, Salam RA, Haider BA, Bhutta ZA. Folic acid supplementation during pregnancy for maternal health and pregnancy outcomes. Cochrane Database Syst Rev. 2013;(3):CD006896.

26 Haider BA, Bhutta ZA. Multiple-micronutrient supplementation for women during pregnancy. Cochrane Database Syst Rev. 2017; 4(4):CD004905.

27 De-Regil LM, Palacios C, Kostiuk LK, Peña-Rosas JP. Vitamin D supplementation for women during pregnancy. Cochrane Database Syst Rev. 2016 Jan 14;(1):CD008873.

28 Middleton P, Gomersall JC, Gould JF, Shepherd E, Olsen SF, Makrides M. Omega-3 fatty acid addition during pregnancy. Cochrane Database Syst Rev. 2018;11(11):CD003402.

29 Ota E, Mori R, Middleton P, Tobe-Gai R, Mahomed K, Miyazaki C, et al. Zinc supplementation for improving pregnancy and infant outcome. Cochrane Database Syst Rev. 2015 Feb 2;2015(2): CD000230

30 Mithal A, Wahl DA, Bonjour JP, Burckhardt P, Dawson-Hughes B, Eisman JA, et al. Global vitamin D status and determinants of hypovitaminosis D. Osteoporos Int. 2009;20(11):1807-20.

31 Gravett MG. Causes of preterm delivery. Semin Perinatol. 1984; $8(4): 246-57$.

32 Karim SM. The role of prostaglandins in human parturition. Proc $\mathrm{R}$ Soc Med. 1971;64(1):10-2.

33 Brazle AE, Johnson BJ, Webel SK, Rathbun TJ, Davis DL. Omega-3 fatty acids in the gravid pig uterus as affected by maternal supplementation with omega-3 fatty acids. J Anim Sci. 2009;87(3):9941002.

34 Ramsden CE, Makrides M, Yuan Z-X, Horowitz MS, Zamora D, Yelland LN, et al. Plasma oxylipins and unesterified precursor fatty acids are altered by DHA supplementation in pregnancy: can they help predict risk of preterm birth? Prostaglandins Leukot Essent Fatty Acids. 2020;153:102041.

35 Meyer BJ, Mann NJ, Lewis JL, Milligan GC, Sinclair AJ, Howe PR. Dietary intakes and food sources of omega- 6 and omega- 3 polyunsaturated fatty acids. Lipids. 2003;38(4):391-8. 
36 Makrides M, Duley L, Olsen SF. Marine oil, and other prostaglandin precursor, supplementation for pregnancy uncomplicated by pre-eclampsia or intrauterine growth restriction. Cochrane Database Syst Rev. 2006;3(3):CD003402.

37 Makrides M, Gibson RA, McPhee AJ, Yelland L, Quinlivan J, Ryan $P$, et al. Effect of DHA supplementation during pregnancy on maternal depression and neurodevelopment of young children: a randomized controlled trial. JAMA. 2010;304(15):1675-83.

38 Zhou SJ, Best K, Gibson R, McPhee A, Yelland L, Quinlivan J, et al. Study protocol for a randomised controlled trial evaluating the effect of prenatal omega-3 LCPUFA supplementation to reduce the incidence of preterm birth: the ORIP trial. BMJ Open. 2017;7(9): e018360.

39 Makrides M, Best K, Yelland L, McPhee A, Zhou S, Quinlivan J, et al. A randomized trial of prenatal $n-3$ fatty acid supplementation and preterm delivery. N Engl J Med. 2019;381(11):1035-45.
40 Simmonds LASTR, Skubisz M, Middleton PF, Best KP, Yelland LN, Quinlivan J, et al. Omega-3 fatty acid supplementation in pregnancy: baseline omega-3 status and early preterm birth: exploratory analysis of a randomised controlled trial. BJOG. $2020 \mathrm{Jul}$; 127:975-81.

41 Olsen SF, Halldorsson TI, Thorne-Lyman AL, Strøm M, Gørtz S, Granstrøm C, et al. Plasma concentrations of long chain N-3 fatty acids in early and mid-pregnancy and risk of early preterm birth. EBioMedicine. 2018;35:325-33.

42 Klebanoff MA, Harper M, Lai Y, Thorp J Jr., Sorokin Y, Varner MW, et al. Fish consumption, erythrocyte fatty acids, and preterm birth. Obstet Gynecol. 2011;117(5):1071-7.

43 Mulholland CA, Benford DJ. What is known about the safety of multivitamin-multimineral supplements for the generally healthy population? Theoretical basis for harm. Am J Clin Nutr. 2007; 85(1):318S-22S. 